\title{
FRACTIONAL EULER METHOD; AN EFFECTIVE TOOL FOR SOLVING FRACTIONAL DIFFERENTIAL EQUATIONS
}

\author{
Hoda F. Ahmed \\ Department of Mathematics, Faculty of Science, Minia University, 61519 Minia, Egypt \\ E-mail: hodamina@yahoo.com
}

Received 24/1/2017

Revised 11/7/2017

Accepted 25/9/2017

\begin{abstract}
Through this article, a numerical scheme based upon the modified fractional Euler method (MFEM) is introduced to find the numerical solutions of linear and nonlinear systems of fractional differential equations (SFDEs) as well as nonlinear multi-order fractional differential equations (MOFDEs). The fractional derivatives are defined by Caputo. The proposed algorithm is very simple and provides the solutions directly without linearization, perturbations or any other assumptions. Illustrating examples with numerical comparisons between the proposed algorithm and the exact and/or fourth order Runge Kutta method (RK4) are given to reveal the efficiency and the accuracy of our algorithm.

Keywords: Systems of nonlinear fractional differential equations, fractional Euler method, multi order fractional differential equations, fractional trapezoidal rule, Caputo fractional derivative.
\end{abstract}

MSC 2010: 65L05. 26A33

\section{Introduction}

In recent decades, fractional differential equations (FDEs) have received significant importance because of their wide-range of uses. Also, several problems in physics, biology, chemistry, applied science and engineering are mathematically modeled by systems of ordinary and fractional differential equations [1-8].

Finding the analytical and/or numerical approximate solutions of different types of FDEs became an exciting topic for many researches [9-11]. Different numerical and analytical techniques have been investigated and developed for solving FDEs, especially the nonlinear problems since most of these equations don't have exact solutions. These techniques include: Adomain decomposition method [12-13], variational iteration method [14], homotopy perturbation method [15], homotopy analysis method [16], fractional differential transform [17] and spectral collocation methods [18].

In this paper, by extending the idea of [19] we construct the solutions of:

(i) The SFDEs:

$D^{\alpha_{i}} x_{i}(t)=f_{i}\left(t, x_{1}, x_{2}, \cdots, x_{n}\right), i=1,2, \ldots, n$

where $D^{\alpha_{i}}$ is the Caputo fractional derivative (CFD) of order

$\alpha_{i}$ and $0<\alpha_{i} \leq 1$, with the initial values

$x_{i}(0)=d_{i}$

(ii) The MOFDEs:

$D^{\alpha} y(t)=F\left(t, y(t), D^{\gamma_{1}} y(t), \cdots D^{\gamma_{m}} y(t)\right), t \in[0,1)$

with the initial conditions

$y^{(k)}(0)=d_{k}, \quad k=0,1, \cdots, m$

where $\quad m<\alpha \leq m+1=\lceil\alpha\rceil, 0<\gamma_{1}<\gamma_{2}<\cdots<\gamma_{m}<\alpha$, $m \in \mathbb{N}$ and $D^{\alpha}$ denote the CFD of order $\alpha$ and $F$ is a nonlinear function.

The existence and uniqueness for SFDEs (1-1) subject to (1-2) and MOFDEs (1-3) subject to (1-4) have been proved in [20] and [21], respectively.

The proposed scheme leads to accurate solutions for the linear and nonlinear operator equations. The analogous solutions of the integer order-equations are considered as special cases of those of fractional order equations.
The structure of this paper is as follows: In the next section, some basic definitions and mathematical preliminaries of the fractional calculus, fractional Euler method for solving initial value FDEs and the modified trapezoidal rule for approximating fractional integrals are introduced. In section 3, the suggested algorithm of MFEM for solving SFDEs is presented with some numerical applications. In section 4 , the procedures of solution of the nonlinear MOFDEs are given with some illustrating examples. Finally the conclusions appear in section 5.

\section{Preliminaries and Notations}

\subsection{Caputo fractional derivatives}

\section{Definition (2-1)}

A real function $f(t), t>0$, is said to be in the space $C_{\mu,} \mu \in \mathcal{R}$, if there exists a real number $p>\mu$ such that $f(t)=t^{p} g(t)$ where $g(t) \in C(0, \infty)$, and is said to be in space $C_{\mu}^{m}$ if and only if $f^{(m)} \in C_{\mu}, m \in \mathbb{N}$.

\section{Definition (2-2)}

The Riemann Liouville fractional integral of order $\alpha \geq 0$ of a function $f \in C_{\mu}, \mu \geq-1$ is defined as

$$
\begin{gathered}
J^{\alpha} f(t)=\frac{1}{\Gamma(\alpha)} \int_{0}^{t}(t-s)^{\alpha-1} f(s) d s, \quad \alpha>0, \quad t>0, \\
J^{0} f(t)=f(t) .
\end{gathered}
$$

It has the properties that: for $f \in C_{\mu}, \mu \in \mathcal{R}, \mu \geq-1, \alpha, \beta \geq$ 0 and $\gamma>-1$
(1) $J^{\alpha} J^{\beta} f(t)=J^{\alpha+\beta} f(t)$
(2) $J^{\alpha} J^{\beta} f(t)=J^{\beta} J^{\alpha} f(t)$
(3) $J^{\alpha} t^{\gamma}=\frac{\Gamma(\gamma+1)}{\Gamma(\gamma+\alpha+1)} t^{\gamma+\alpha}$

For more details see [10].

Definition (2-3)

The CFD, $D^{\alpha}$, of $f(t)$ is defined as

$$
D^{\alpha} f(t)=\frac{1}{\Gamma(m-\alpha)} \int_{0}^{t}(t-s)^{m-\alpha-1} f^{(m)}(s) d s
$$

For $m-1<\alpha \leq m, m \in \mathbb{N}, t>0, f(t) \in C_{-1}^{m}$.

For more details about fractional derivatives, see [11]. 
Lemma1. If $m-1<\alpha \leq m, m \in \mathbb{N}$ and $f \in C_{\mu}^{m}, \mu \geq-1$, then

$$
\begin{gathered}
D^{\alpha} J^{\alpha} f(t)=f(t) \\
J^{\alpha} D^{\alpha} f(t)=f(t)-\sum_{k=0}^{m-1} f^{(k)}\left(0^{+}\right) \frac{t^{k}}{k !}, \quad x>0 .
\end{gathered}
$$

\subsection{Fractional Euler's method}

Consider the initial value problem

$D^{\alpha} y(t)=f(t, y(t)), y(0)=y_{0}, 0<\alpha \leq 1, t>0$.

To find the solution of (2-3) over the interval $[0, a]$, a set of points $\left\{\left(t_{j}, y\left(t_{j}\right)\right\}\right.$ are produced which are used as approximated values. In order to achieve this approximation, the interval $[0, a]$ is partitioned into $n$ subintervals $\left[t_{j}, t_{j+1}\right]$ each of equal width $h=\frac{a}{n}, t_{j}=j h$ for $j=0,1, \cdots, n$.

The general formula for the fractional Euler method is

$$
\begin{aligned}
& y\left(t_{j+1}\right)=y\left(t_{j}\right)+\frac{h^{\alpha}}{\Gamma(\alpha+1)} f\left(t_{j}, y\left(t_{j}\right)\right), \\
& t_{j+1}=t_{j}+h, \quad j=0,1, \cdots, n-1 .
\end{aligned}
$$

For more details, see [19].

\subsection{Modified trapezoidal rule}

The modified trapezoidal rule is introduced in [22]. This is used for approximating the fractional integral of a function $f(t) \in$ $C^{2}[0, a], J^{\alpha} f(t)$, by a weighted sum of function values at identified points. Supposing that the interval $[0, a]$ is partitioned into $n$ subintervals each of length $h=\frac{a}{n}$ by using the nodes $t_{j}=$ $j h, j=0,1,2, \ldots, n$.

general the modified trapezoidal rule $T(f, h, \alpha)=\left((n-1)^{\alpha+1}-(n-\alpha-1) n^{\alpha}\right) \frac{h^{\alpha} f(0)}{\Gamma(\alpha+2)}+\frac{h^{\alpha} f(a)}{\Gamma(\alpha+2)}+$

$\sum_{j=1}^{n-1}\left((n-j+1)^{\alpha+1}-2(n-j)^{\alpha+1}+(n-j-1)^{\alpha+1}\right) \frac{h^{\alpha} f\left(t_{j}\right)}{\Gamma(\alpha+2)}$,

(2-6)

is an approximation to the fractional integral

$\left(J^{\alpha} f(t)\right)(a)=T(f, h, \alpha)-E_{T}(f, h, \alpha), \quad a>0, \quad \alpha>0$.

With the error, $E_{T}(f, h, \alpha)$, defined by

$$
\left|E_{T}(f, h, \alpha)\right| \leq \omega_{\alpha}\left\|f^{\prime \prime}\right\|_{\infty} a^{\alpha} h^{2}=O\left(h^{2}\right),
$$

where $\omega_{\alpha}$ is a constant depends on $\alpha$ only.

\section{The Proposed Algorithm for Solving SFDEs}

Consider the SFDEs:

$D^{\alpha_{i}} x_{i}(t)=f_{i}\left(t, x_{1}, x_{2}, \cdots, x_{n}\right), i=1,2, \ldots, n$

where $D^{\alpha_{i}}$ is the CFD of order $\alpha_{i}$ and $0<\alpha_{i} \leq 1$, with the initial values: $x_{i}(0)=d_{i}$,

The proposed algorithm consists of the following steps:

(i) Transforming Eq. (3-1) into an integral equation; by applying the fractional integral operator $J^{\alpha_{i}}$ to Eq. (3-1) yields

$$
x_{i}(t)=J^{\alpha_{i}} f_{i}\left(t, x_{1}, x_{2}, \cdots, x_{n}\right)+d_{i}
$$

(ii) Dividing the interval $[0, a]$ into $n$ subintervals $\left[t_{j}, t_{j+1}\right]$ each of width $h=\frac{a}{n}, t_{j}=j h$ for $j=0,1, \cdots, n$.

(iii) Substituting $t=t_{1}$ in Eq. (3-2) gives the solution at the point $\left(t_{1}, x_{i}\left(t_{1}\right)\right)$ as $\quad x_{i}\left(t_{1}\right)=$ $J^{\alpha_{i}} f_{i}\left(t, x_{1}, x_{2}, \cdots, x_{n}\right)\left(t_{1}\right)+d_{i}$

(iv) Applying the modified trapezoidal rule (2-6) to approximate the term $J^{\alpha_{i}} f_{i}\left(t, x_{1}, x_{2}, \cdots, x_{n}\right)\left(t_{1}\right)$ with step size $h=t_{1}-t_{0}, \quad$ gives $\quad x_{i}\left(t_{1}\right)=$ $\alpha_{i} \frac{h^{\alpha_{i}} f_{i}\left(t_{0}, d_{1}, d_{2}, \cdots, d_{n}\right)}{\Gamma\left(\alpha_{i}+2\right)}+\frac{h^{\alpha_{i}} f_{i}\left(t_{1}, x_{1}\left(t_{1}\right), x_{2}\left(t_{1}\right), \cdots, x_{n}\left(t_{1}\right)\right)}{\Gamma\left(\alpha_{i}+2\right)}+d_{i}$. $(3-4)$

(v) Approximating $x_{i}\left(t_{1}\right)$ in Eq. (3-4) by using the fractional Euler method (2-4) yields

$$
\begin{aligned}
& x_{i}\left(t_{1}\right)=\alpha_{i} \frac{h^{\alpha_{i}} f_{i}\left(t_{0}, d_{1}, d_{2}, \cdots, d_{n}\right)}{\Gamma\left(\alpha_{i}+2\right)}+h^{\alpha_{i}} f_{i}\left(t_{1}, d_{1}+\right. \\
& \frac{h^{\alpha_{i}}}{\Gamma\left(\alpha_{i}+1\right)} f_{1}\left(t_{0}, x_{1}\left(t_{0}\right), \cdots, x_{n}\left(t_{0}\right)\right), \cdots, d_{n}+ \\
& \left.\frac{h^{\alpha_{i}}}{\Gamma\left(\alpha_{i}+1\right)} f_{n}\left(t_{0}, x_{1}\left(t_{0}\right), x_{2}\left(t_{0}\right), \cdots, x_{n}\left(t_{0}\right)\right)\right) / \Gamma\left(\alpha_{i}+2\right)+d_{i} .
\end{aligned}
$$

Repeat the process to generate a sequence of points which approximate the solutions $x_{i}(t), i=1,2, \ldots, n$.

So the explicit formula for our scheme is

$$
\begin{aligned}
& x_{i}\left(t_{j}\right)=\frac{h^{\alpha_{i}}}{\Gamma\left(\alpha_{i}+2\right)}\left((j-1)^{\alpha_{i}+1}-\left(j-\alpha_{i}-\right.\right. \\
& \left.1) j^{\alpha_{i}}\right) f_{i}\left(t_{0}, d_{1}, d_{2}, \cdots, d_{n}\right)+d_{i}+\frac{h^{\alpha_{i}}}{\Gamma\left(\alpha_{i}+2\right)} \sum_{k=1}^{j-1}((j-k+ \\
& 1)^{\alpha_{i}+1}-2(j-k)^{\alpha_{i}+1}+(j-k- \\
& \left.1)^{\alpha_{i}+1}\right) f_{i}\left(t_{k}, x_{1}\left(t_{k}\right), \cdots, x_{n}\left(t_{k}\right)\right)+\frac{h^{\alpha_{i}}}{\Gamma\left(\alpha_{i}+2\right)} f_{i}\left(t_{j}, x_{1}\left(t_{j-1}\right)+\right. \\
& \frac{h^{\alpha_{i}}}{\Gamma\left(\alpha_{i}+1\right)} f_{1}\left(t_{j-1}, x_{1}\left(t_{j-1}\right), \cdots, x_{n}\left(t_{j-1}\right)\right), \cdots, x_{n}\left(t_{j-1}\right)+ \\
& \left.\frac{h^{\alpha_{i}}}{\Gamma\left(\alpha_{i}+1\right)} f_{n}\left(t_{j-1}, x_{1}\left(t_{j-1}\right), \cdots, x_{n}\left(t_{j-1}\right)\right)\right) .
\end{aligned}
$$

\subsection{Numerical applications}

In this subsection three SFDEs are solved by the proposed technique one of them is linear and the two others are nonlinear.

\section{Example (3-1) (linear SFDEs)}

$$
\begin{array}{cl}
D^{\alpha} x(t)=x(t)+y(t), & 0<\alpha \leq 1 \\
D^{\beta} y(t)=-x(t)+y(t), & 0<\beta \leq 1 .
\end{array}
$$

with the initial values: $x(0)=0, \quad y(0)=1$.

At $\alpha=\beta=1$, the fractional system (3-5) has exact solution $\mathrm{x}(\mathrm{t})=e^{t} \sin (t)$ and $\mathrm{y}(\mathrm{t})=e^{t} \cos (t)$.

According to the suggested methodology, the numerical solution to system (3-5) in the interval $[0,1]$ with fixed step size $h=0.01$ is graphically illustrated in Figure 1 and tabulated in Table 1
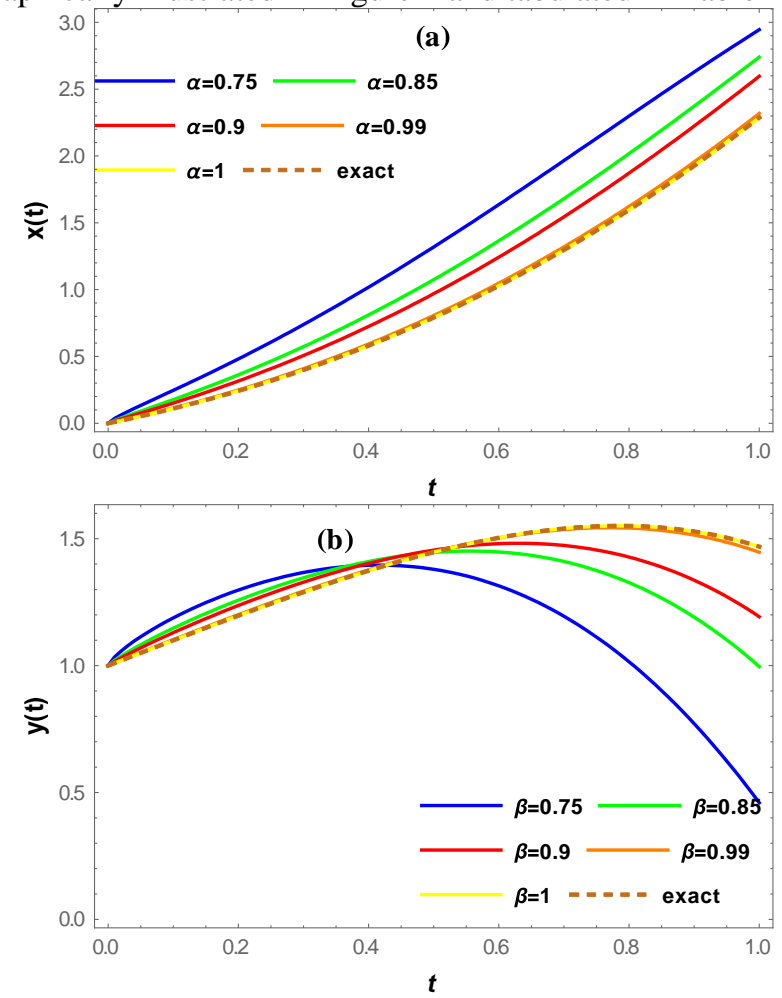

Fig.1 Behaviour of $x(t)$ and $y(t)$ by MFEM for various values of $\alpha, \beta$ with exact solutions for example (3-1).

Figure 1 (a) and (b) reveal the behavior of estimated solutions of $x(t)$ and $y(t)$, respectively by MFEM attained at various values of $\alpha$ and $\beta(\alpha=\beta=0.75,0.85,0.9,0.99$ and 1) in comparisons with the exact solutions at $\alpha=\beta=1$. Table 1 displays the 
absolute errors between the approximated results obtained by

MFEM and the exact solutions.

Example (3-2) (nonlinear SFDEs)

$$
\begin{aligned}
D^{\alpha} x(\mathrm{t}) & =-x(\mathrm{t}), \\
D^{\alpha} y(\mathrm{t}) & =x(\mathrm{t})-y^{2}(\mathrm{t}), \\
D^{\alpha} z(\mathrm{t}) & =y^{2}(\mathrm{t}),
\end{aligned}
$$

with $0<\alpha \leq 1$ and initial values $x(0)=1, y(0)=0, z(0)=0$. According to the suggested methodology, the numerical solution to system (3-6) in the interval $[0,1]$ with fixed step size $h=0.01$ are graphically illustrated in Figure 2 and tabulated in Table 2.
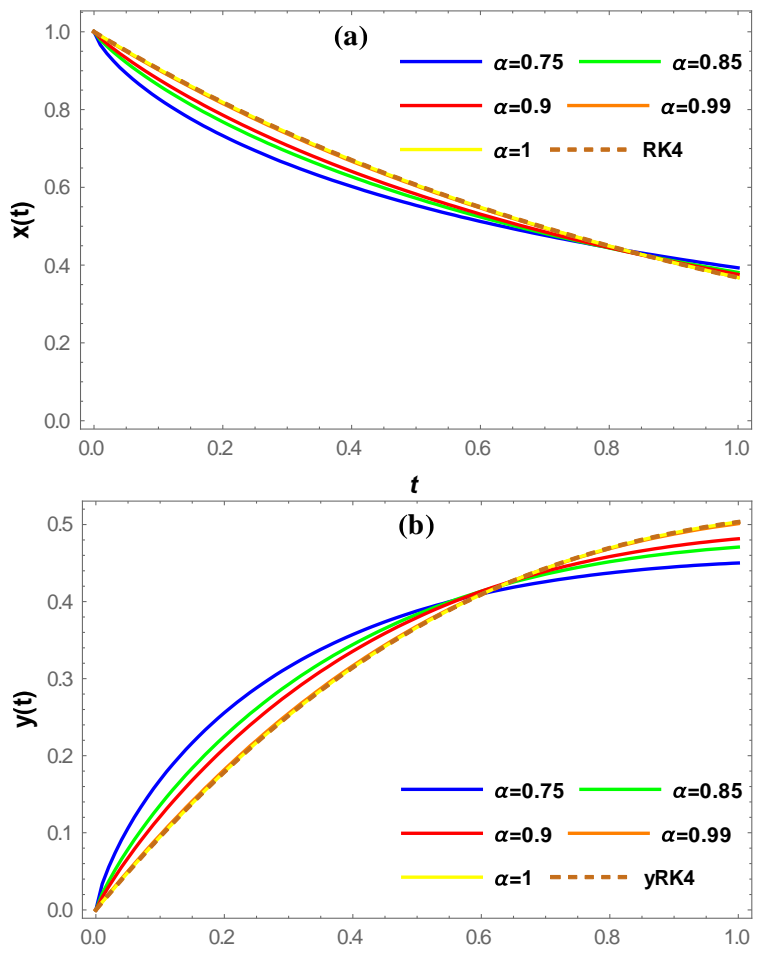

\begin{tabular}{|c|c|c|c|c|c|c|c|c|c|}
\hline & \multicolumn{3}{|c|}{$x(t)$} & \multicolumn{3}{|c|}{$y(t)$} & \multicolumn{3}{|c|}{$\mathrm{z}(\mathrm{t})$} \\
\hline $\mathrm{t}$ & $\mathrm{x}_{\text {MFEM }}$ & $\mathrm{X}_{\mathrm{RK} 4}$ & $\left|\mathrm{x}_{\mathrm{RK} 4}-\mathrm{x}_{\mathrm{MFEM}}\right|$ & $y_{\text {MFEM }}$ & $\mathrm{y}_{\mathrm{RK} 4}$ & $\left|\mathrm{y}_{\mathrm{RK} 4}-\mathrm{y}_{\mathrm{MFEM}}\right|$ & $\mathrm{Z}_{\text {MFEM }}$ & $\mathrm{z}_{\mathrm{RK} 4}$ & $\left|z_{\text {RK4 }}-z_{\text {MFEM }}\right|$ \\
\hline 0. & 1. & 1. & 0 & 0. & 0. & 0 & 0. & 0. & 0 \\
\hline 0.1 & 0.90484 & 0.90484 & $5.4832 \times 10^{-7}$ & 0.094853 & 0.09485 & $9.1178 \times 10^{-7}$ & 0.0003097 & 0.000308 & $1.4601 \times 10^{-6}$ \\
\hline 0.2 & 0.81873 & 0.81873 & $1.199 \times 10^{-6}$ & 0.179 & 0.179 & $1.2089 \times 10^{-6}$ & 0.0022696 & 0.002267 & $2.4079 \times 10^{-6}$ \\
\hline 0.3 & 0.74082 & 0.74082 & $1.7209 \times 10^{-6}$ & 0.25218 & 0.25218 & $1.1721 \times 10^{-6}$ & 0.0070022 & 0.006999 & $2.893 \times 10^{-6}$ \\
\hline 0.4 & 0.67032 & 0.67032 & $2.1326 \times 10^{-6}$ & 0.31457 & 0.31457 & $8.7637 \times 10^{-7}$ & 0.015115 & 0.015112 & $3.0089 \times 10^{-6}$ \\
\hline 0.5 & 0.60653 & 0.60653 & $2.4503 \times 10^{-6}$ & 0.36668 & 0.36668 & $4.1659 \times 10^{-7}$ & 0.026796 & 0.026793 & $2.8669 \times 10^{-6}$ \\
\hline 0.6 & 0.54881 & 0.54881 & $2.6883 \times 10^{-6}$ & 0.40927 & 0.40927 & $1.1527 \times 10^{-7}$ & 0.041922 & 0.04192 & $2.573 \times 10^{-6}$ \\
\hline 0.7 & 0.49658 & 0.49659 & $2.8588 \times 10^{-6}$ & 0.44326 & 0.44326 & $6.4376 \times 10^{-7}$ & 0.060159 & 0.060157 & $2.215 \times 10^{-6}$ \\
\hline 0.8 & 0.44933 & 0.44933 & $2.9725 \times 10^{-6}$ & 0.46962 & 0.46962 & $1.1157 \times 10^{-6}$ & 0.081053 & 0.081051 & $1.8568 \times 10^{-6}$ \\
\hline 0.9 & 0.40657 & 0.40657 & $3.0386 \times 10^{-6}$ & 0.48934 & 0.48934 & $1.4997 \times 10^{-6}$ & 0.10409 & 0.10409 & $1.5389 \times 10^{-6}$ \\
\hline 1. & 0.36788 & 0.36788 & $3.0653 \times 10^{-6}$ & 0.50335 & 0.50335 & $1.7826 \times 10^{-6}$ & 0.12878 & 0.12877 & $1.2827 \times 10^{-6}$ \\
\hline
\end{tabular}

\begin{tabular}{|c|c|c|c|c|c|c|}
\multicolumn{1}{|c|}{$\boldsymbol{x}(\mathrm{t})$} & \multicolumn{3}{|c|}{$\mathrm{y}(\mathrm{t})$} \\
\hline & \multicolumn{7}{|c|}{$\mathrm{x}_{\text {exact }}$} & $\left|\mathrm{x}_{\text {exact }}-\mathrm{x}_{\text {MFEM }}\right|$ & $\mathrm{y}_{\text {MFEM }}$ & $\mathrm{y}_{\text {exact }}$ & $\left|\mathrm{y}_{\text {exact }}-\mathrm{y}_{\text {MFEM }}\right|$ \\
\hline $\mathrm{t}$ & $\mathrm{x}_{\text {MFEM }}$ & 0. & 0. & 1. & 1. & 0. \\
0.1 & 0.110334 & 0.11033 & $1.16344 \times 10^{-6}$ & 1.09965 & 1.09965 & $1.31325 \times 10^{-6}$ \\
0.2 & 0.242658 & 0.24266 & $2.74861 \times 10^{-6}$ & 1.19705 & 1.19706 & $3.85281 \times 10^{-6}$ \\
0.3 & 0.398915 & 0.39891 & $4.1208 \times 10^{-6}$ & 1.28956 & 1.28957 & $7.22567 \times 10^{-6}$ \\
0.4 & 0.580949 & 0.580944 & $5.11306 \times 10^{-6}$ & 1.37405 & 1.37406 & 0.0000115217 \\
0.5 & 0.790445 & 0.790439 & $5.52321 \times 10^{-6}$ & 1.44687 & 1.44689 & 0.0000168185 \\
0.6 & 1.02885 & 1.02885 & $5.1111 \times 10^{-6}$ & 1.50384 & 1.50386 & 0.0000231749 \\
0.7 & 1.2973 & 1.2973 & $3.59642 \times 10^{-6}$ & 1.54017 & 1.5402 & 0.0000306235 \\
0.8 & 1.59651 & 1.59651 & $6.5729 \times 10^{-7}$ & 1.55051 & 1.55055 & 0.0000391618 \\
0.9 & 1.92667 & 1.92667 & $4.07029 \times 10^{-6}$ & 1.52887 & 1.52891 & 0.0000487429 \\
1. & 2.28734 & 2.28736 & 0.0000109917 & 1.46863 & 1.46869 & 0.0000592637 \\
\hline
\end{tabular}

Table1.The absolute errors of example (3-1) between the approximate values by MFEM and the exact solutions

\begin{tabular}{|c|c|c|c|c|c|c|c|c|c|}
\hline & \multicolumn{3}{|c|}{$x(t)$} & \multicolumn{3}{|c|}{$\mathrm{y}(\mathrm{t})$} & \multicolumn{3}{|c|}{$\mathrm{z}(\mathrm{t})$} \\
\hline $\mathrm{t}$ & $\mathrm{X}_{\text {MFEM }}$ & $\mathrm{X}_{\mathrm{RK} 4}$ & $\left|\mathrm{x}_{\mathrm{RK} 4}-\mathrm{x}_{\mathrm{MFEM}}\right|$ & $\mathrm{y}_{\text {MFEM }}$ & $\mathrm{y}_{\mathrm{RK} 4}$ & $\left|\mathrm{y}_{\mathrm{RK} 4}-\mathrm{y}_{\mathrm{MFEM}}\right|$ & $\mathrm{z}_{\text {MFEM }}$ & $\mathrm{Z}_{\mathrm{RK} 4}$ & $\left|\mathrm{z}_{\mathrm{RK} 4}-\mathrm{z}_{\mathrm{MFEM}}\right|$ \\
\hline
\end{tabular}

Table2.The absolute errors of example (3-2) between the approximate values obtained by MFEM and RK4 at $\alpha=1$
$D^{\alpha} z(t)=y(t) z(t)$

$\alpha \leq 1$.

By using the suggested methodology, the numerical solution to system (3-7) in the interval $[0,1]$ with fixed step size $h=0.01$ are graphically illustrated in Figure 3 and tabulated in Table 3. 


\begin{tabular}{|c|c|c|c|c|c|c|c|c|c|}
\hline 0. & 0. & 0. & 0. & 1. & 1. & 0. & 1. & 1. & 0. \\
0.1 & 0.20007 & 0.20007 & $9.5387 \times 10^{-7}$ & 1.0007 & 1.0007 & $3.3364 \times 10^{-6}$ & 1.1052 & 1.1052 & $8.9185 \times 10^{-7}$ \\
0.2 & 0.40107 & 0.40107 & $4.5822 \times 10^{-6}$ & 1.0053 & 1.0053 & $6.7393 \times 10^{-6}$ & 1.2217 & 1.2217 & $3.1868 \times 10^{-6}$ \\
0.3 & 0.60545 & 0.60544 & 0.000011075 & 1.0181 & 1.0181 & 0.000010412 & 1.3517 & 1.3517 & $7.1596 \times 10^{-6}$ \\
0.4 & 0.8174 & 0.81738 & 0.000020921 & 1.0431 & 1.0431 & 0.000014729 & 1.4983 & 1.4982 & 0.000013588 \\
0.5 & 1.0432 & 1.0432 & 0.000035167 & 1.0851 & 1.0851 & 0.00002028 & 1.6662 & 1.6662 & 0.000023709 \\
0.6 & 1.292 & 1.292 & 0.000055794 & 1.1494 & 1.1494 & 0.000027989 & 1.8628 & 1.8628 & 0.000039598 \\
0.7 & 1.577 & 1.5769 & 0.000086418 & 1.2427 & 1.2426 & 0.00003933 & 2.0989 & 2.0988 & 0.000064906 \\
0.8 & 1.9177 & 1.9175 & 0.00013365 & 1.3736 & 1.3735 & 0.000056759 & 2.3914 & 2.3913 & 0.00010638 \\
0.9 & 2.3441 & 2.3439 & 0.00020991 & 1.5543 & 1.5543 & 0.000084555 & 2.7671 & 2.767 & 0.00017719 \\
1. & 2.9043 & 2.9039 & 0.00033971 & 1.8028 & 1.8027 & 0.00013058 & 3.2708 & 3.2705 & 0.0003049 \\
\hline
\end{tabular}

Table3.The absolute errors of example (3-3) between the approximate values obtained MFEM and RK4 solutions at $\alpha=1$
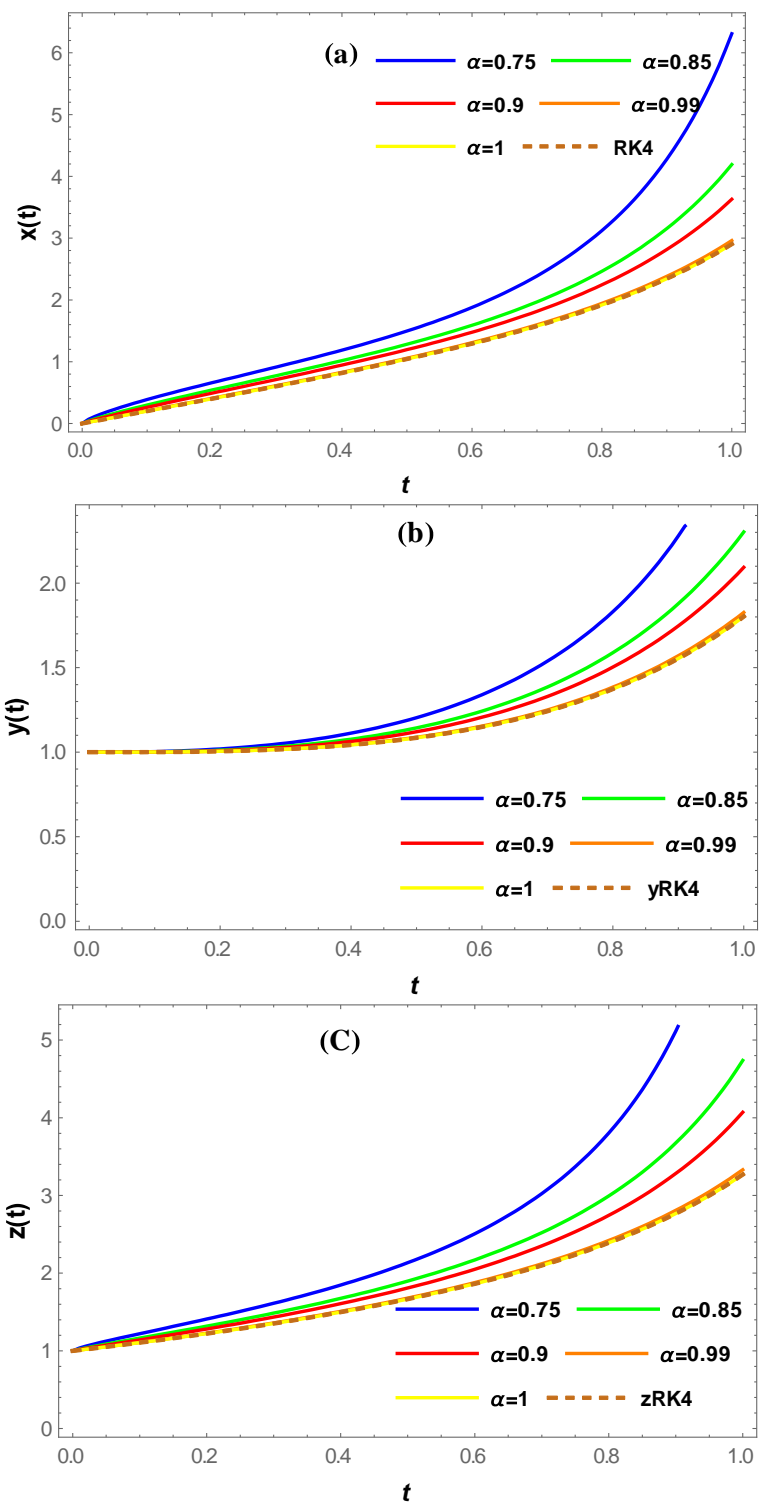

Fig.3. Behavior of approximate solution by MFEM for different values of $\alpha$ with RK4 at $\alpha=1$ of example3

The numerical results for example (3-3) are shown graphically by Figures 3. Figures 3 (a), (b) and (c) display the behavior of estimated results of $x(t), y(t)$ and $z(t)$, respectively obtained by MFEM for various values of $\alpha(\alpha=0.75,0.85,0.9,0.99$ and 1$)$ and $h=0.01$ in comparisons with the solutions given by RK4 for $x(t), y(t)$ and $z(t)$, respectively at $\alpha=1, h=0.01$.

The absolute errors of example (3-3) between the approximated solutions given by MFEM and the RK4 method at $\alpha=1$ and $h=0.01$ are listed in Table 3 .

\section{Procedure Solution to MOFDEs}

In order to apply our methodology to MOFDE; at first the MOFDE is transformed into an equivalent system of FDEs of a single order as discussed in the following subsection.

\subsection{Convert MOFDE into a system of FDEs}

Consider the following MOFDE

$D^{\alpha} y(t)=F\left(t, y(t), D^{\gamma_{1}} y(t), \cdots D^{\gamma_{m}} y(t)\right), t \in[0,1)$.

Where, $m<\alpha \leq m+1, m \in \mathbb{N}, 0<\gamma_{1}<\gamma_{2}<\cdots \leq \gamma_{m}<\alpha$.

Eq. (4-1) can be converted into a system of FDEs as follows:

Let $y_{1}=y$ and assume $D^{\gamma_{1}} y_{1}=y_{2}$,

Case (i)

If $m-1 \leq \gamma_{1}<\gamma_{2} \leq m$, then assume $D^{\gamma_{2}-\gamma_{1}} y_{2}=y_{3}$.

Case (ii)

Consider $m-1 \leq \gamma_{1}<m \leq \gamma_{2}$. If $\gamma_{1}=m-1$, then assume $D^{\gamma_{2}-\gamma_{1}} y_{2}=y_{3}$.

If $m-1<\gamma_{1}<m \leq \gamma_{2}$, then assume $D^{m-\gamma_{1}} y_{2}=y_{3}$.

Similar steps can be transformed Eq. (4-1) to a system of FDEs.

Example (4-1) Consider the FDE

$D^{2} y(t)+D^{\frac{3}{2}} y(t)+y(t)=t^{2}+2+\frac{\Gamma(3)}{\Gamma\left(\frac{3}{2}\right)} t^{\frac{1}{2}}$

With the initial conditions: $\mathrm{y}(0)=0, \mathrm{y}^{\prime}(0)=0$,

which has the exact solution: $\mathrm{y}(\mathrm{t})=\mathrm{t}^{2}$.

According to our proposed method, by changing variable $y_{1}(x)=$ $y(x)$, Eq.(4-2) is transformed into the following system of FDEs

$$
\begin{gathered}
D^{\frac{1}{2}} y_{1}(t)=y_{2}(t) \\
D^{\frac{1}{2}} y_{2}(t)=y_{3}(t) \\
D^{\frac{1}{2}} y_{3}(t)=y_{4}(t) \\
D^{\frac{1}{2}} y_{4}(t)=-y_{4}(t)-y_{1}(t)+t^{2}+2+\frac{\Gamma(3)}{\Gamma\left(\frac{3}{2}\right)} t^{\frac{1}{2}}
\end{gathered}
$$

with the initial values:

$y_{1}(0)=y(0), y_{2}(0)=y(0), y_{3}(0)=y(0), y_{4}(0)=0$.

According to MFEM the approximated solution to system (4-2) in the interval $[0,1]$ with fixed step sizes $(h=$ $0.01,0.005$ and 0.001 ) and the exact solution are graphically illustrated in Figure 4

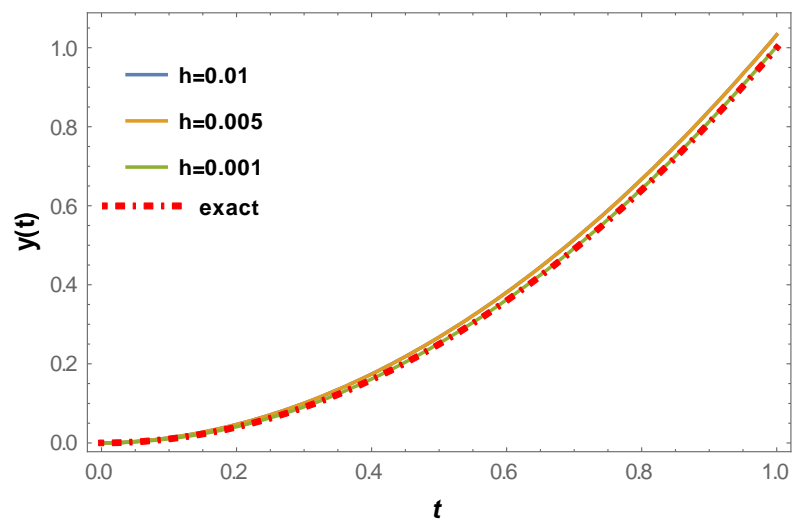


Fig.4 Comparison between the exact solution and the proposed algorithm for different values $\mathrm{f} h(h=0.01,0.005$ and 0.001$)$ for example (4-1)

Example (4-2) Consider Bagley-Torvik equation [23]

$A D^{2} y(t)+B D^{\frac{3}{2}} y(t)+C y(t)=f(t)$

with $\mathrm{y}(0)=0$, y $(0)=1,0 \leq \mathrm{t} \leq 5$

where $A=M, B=2 S \sqrt{\mu \rho}$ and $C=K$.

This equation depicts the dynamics of a large plate with surface $S$ and mass $M$ in a Newtonian fluid. The fluid has viscosity $\mu$ and density $\rho$. The plate is hanged on a massless spring of stiffness $K$. In Equation (4-3), $f(t)$ describes a loading force. We will find the solution of Eq. (4-3) under the assumptions of $A=B=C=1$ and $f(t)=C(1+t)$.

Eq. (4-3) has exact solution: $y(t)=(1+t)$.

According to our procedure Eq. (4-3) is converted into a system of FDEs by changing variable $y_{1}(t)=y(t)$ getting

$$
\begin{gathered}
D^{\frac{1}{2}} y_{1}(t)=y_{2}(t) \\
D^{\frac{1}{2}} y_{2}(t)=y_{3}(t) \\
D^{\frac{1}{2}} y_{3}(t)=y_{4}(t) \\
D^{\frac{1}{2}} y_{4}(t)=-y_{1}(t)-y_{4}(t)+(t+1),
\end{gathered}
$$

With the initial values:

$$
y_{1}(0)=1, \quad y_{2}(0)=0, \quad y_{3}(0)=1, \quad y_{4}(0)=0
$$

By applying MFEM, the numerical solution to system (4-3) in the interval $[0,5]$ with fixed step sizes $(h=0.05,0.01$ and 0.001$)$ and the exact solution are graphically illustrated in Figure 5.

Figures 4 and 5 demonstrate that the approximated solutions gained by MFEM are approximately coincided with the exact solutions. Also the accurateness of the anticipated technique can be enhanced if we use much smaller value of $h$.

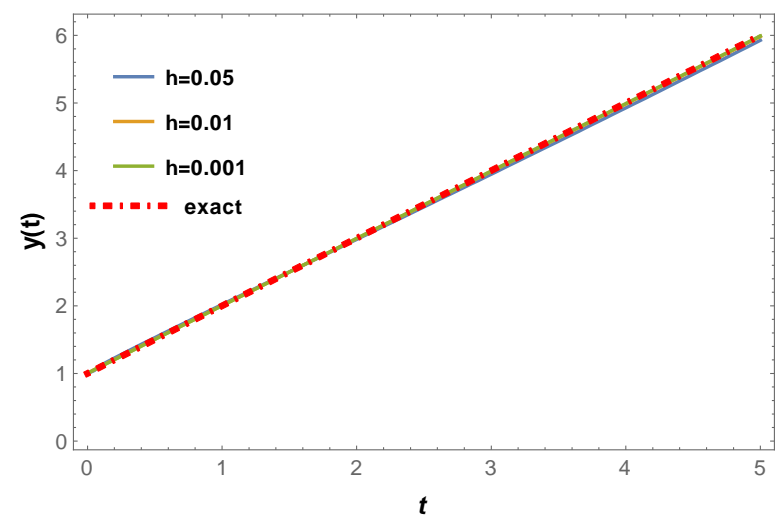

Fig.5 Comparison between the exact solution and the proposed algorithm for different values of $h(h=0.05,0.01$ and 0.001$)$ for example (4-2)

\section{Conclusion}

Through the present paper, the applications of numerical scheme based on the MFEM have been extended to the solution of the linear and nonlinear SFDEs and nonlinear MOFDs. This numerical scheme was used directly without consuming linearization, perturbation or other restrictive assumptions. Illustrative examples are given to certify the applicability of our proposed method. These numerical examples have proved good results when compared with exact solutions or other known numerical methods such as RK4 in the integer order case. It's also noted that the accuracy of the suggested scheme is enhanced as the step size decreases.

\section{Acknowledgements}

The author is very grateful to the referees for carefully reading the paper and for their comments and suggestions which have improved the paper

\section{References}

1. T. M. Atanackovic and B. Stankovic: On a System of Differential Equations with Fractional Derivatives Arising in Rod Theory. J. Phys. A. 2004; 37, 1241-1250.

2. P. L. Butzer and U. Westphal: An Introduction to Fractional Calculus. Ch. 1 in Applications of Fractional Calculus in Physics (Ed. R. Hilfer). Singapore. World Scientific. 2000

3. L. Debnath: Fractional Differential Equations in Fluid Mechanics, J. Frac. Cal., Appl. Anal. 2003.

4. F. Mainardi: Fractional Relaxation in an Elastic Solid. J. Alloys and Compounds (JALCOM). 1994; 94, 534-538.

5. B. Ross: Fractional Calculus and its Applications. Proceedings of the International Conference on Fractional Calculus and its Applications, Univ. of New York, West Haven. Conn. Springer-Verlage, New York, 1975

6. H. M. Srivasta and R. K. Saxena: Operators of Fractional Integration and Their Applications. J. Appl. Math. and Comput. 2001; 118, 1-52.

7. S. Samko, O. Marichev and A. Kikbas: Fractional Integrals and Derivatives and Some of Their Applications. Sci. and Tech. Minsk, Russian, 1987

8. N. Shimizu and W. Zhang: Fractional Calculus Approach to Dynamic Problems of Viscoelastic Materials. (JSME series), C-Mechanical Systems, Machine Elements and Manufacturing. 1999; 42, 825-837

9. V. Daftardar-Gejji and A. Babakhani: Analysis of a System of Fractional Differential Equations. J. Math. Anal. Appl., 2004; 293, 511-522.

10. K. S. Miller and B. Ross: An Introduction to Fractional Calculus and Fractional Differential Equations. John Wiley and Sons, New York. 1993.

11. I. Podlubny: Fractional Differential Equations. Academic Press. San Diego. 1999.

12. H. Jafari and V. D. Gejji: Solving a System of Nonlinear Fractional Differential Equations Using Adomain Decomposition. J. Com. And Appl. Math. 2005.

13. S. S. Ray and R. K. Bera: Analytical Solution of the Bagley Torvik Equation by Adomain Decomposition Method. J. Appl. Math. Comput. 2005; 168, 398 -410

14. J. H. He: Variational Iteration Method Some Recent Results and New Interpretations. J. Comput. Appl. Math. 2007; 207, 3-17.

15. A. A. Hemeda: Homotopy Perturbation Method for Solving Systems of Nonlinear Coupled Equations. Appl. Math. Sci. 2012; 6, 4787-4800.

16. I. Hashim, O. Abdulaziz, S. Momani: Homotopy Analysis Method for Fractional IVPs, Commun. Nonlinear Sci. Numer. Simul. 2009; 14, 674-684.

17. V. S. Ertürk and S. Momani: Solving Systems of Fractional Differential Equations Using Differential Transform Method. J. Comput. Appl. Math. 2008; 215, no. 1, 142-151.

18. E. H. Doha, A.H. Bhrawy, S. S. Ezz-Eldien: A Chebyshev Spectral Method Based on Operational Matrix for Initial and Boundary Value Problems of Fractional Order. Comput. Math. Appl. 2011; 62, 2364-2373.

19. Z. M. Odibat and S. Momani: An Algorithm for the Numerical Solutions of Differential Equations of Fractional Order. J. Appl. Math. \& Informatics. 2008; 26, 15-27.

20. V. Daftardar-Gejji, A. Babakhani: Analysis of a System of Fractional Differential Equations, J. Math. Anal. Appl. 2004; 293, 511-522. 
21. K. Diethelm, N.J. Ford, Multi-Order Fractional Differential Equations and Their Numerical Solutions, Appl. Math. Comput. 2004: 154, 621-640.

22. Z. M. Odibat: Approximation of Fractional Integral and Caputo Fractional Derivatives. J. Appl. Math. Comput. 2006; 178(2), 527-533.

23. S. Momani and K. Al Khaled, Numerical Solutions for Systems of Fractional Differential Equations by the Decomposition Method, J. Appl. Math. Comput. 2005; 162, 1351-1365. 